\title{
Dificuldades da reabilitação cardíaca no Brasil: uma revisão integrativa
}

\author{
Difficulties of cardiac rehabilitation in Brazil: an integrative review \\ Dificultades de la rehabilitación cardíaca en Brasil: una revisión integradora
}

Bárbara Pires Ihara ${ }^{1 *}$, Ana Cláudia Carvalho Prieto ${ }^{1}$, Marlucia do Nascimento Nobre ${ }^{1}$, Angelo Bruno Pagoto ${ }^{1}$, Irineu Lopes de Alcântara Júnior ${ }^{1}$, Armando Hiroyuki Mori Júnior ${ }^{1}$.

\section{RESUMO}

Objetivo: Avaliar as barreiras para a ampla implementação da Reabilitação Cardíaca que impedem a população brasileira de aderir aos programas de tratamento e fomentar medidas públicas para mudar tal realidade. Métodos: Esse estudo é uma revisão de literatura integrativa. Para coleta dos dados foram utilizadas plataformas: Acervo+, Biblioteca Virtual em Saúde (BVS) e Pubmed e os descritores em ciências da Saúde: "reabilitação cardíaca", "Brasil" e "baixa renda" ou "pobreza". Incluiu-se artigos completos em português, inglês e espanhol, entre 2010 e 2021. A coleta foi realizada em cinco etapas: identificação, seleção, elegibilidade, inclusão e síntese, reunindo em fluxogramas e quadros para análise. Resultados: A pesquisa localizou 278 artigos. Após leitura do título e resumo dos artigos foram excluídos 250 repetidos ou atemáticos. Foram selecionados 28 trabalhos para leitura integral. Após análise foram incluídos um total de quinze artigos para a presente revisão. Considerações finais: É fundamental investir em RC visando saúde e qualidade de vida. Para isso, faz-se necessário que estratégias específicas, abordando os principais pontos de dificuldades à implantação da RC sejam elaboradas e colocadas em prática.

Palavras-chave: Reabilitação cardíaca, Brasil, Pobreza.

\begin{abstract}
Objective: Assess the barriers to the wide implementation of Cardiac Rehabilitation that prevent the Brazilian population from adhering to treatment programs and promote public measures to change this reality. Methods: This study is an integrative literature review. For data collection, platforms were used: Collection+, Virtual Health Library (VHL) and Pubmed and the descriptors in Health Sciences: "cardiac rehabilitation", "Brazil" and "low income" or "poverty". Complete articles in Portuguese, English and Spanish were included, between 2010 and 2021. The collection was carried out in five stages: identification, selection, eligibility, inclusion and synthesis, gathering in flowcharts and tables for analysis. Results: The search found 278 articles. After reading the title and abstract of the articles, 250 repeated or athematic articles were excluded. 28 works were selected for full reading. After analysis, a total of fifteen articles were included for this review. Final considerations: It is essential to invest in CR for health and quality of life. For this, it is necessary that specific strategies, addressing the main points of difficulties for the implementation of $\mathrm{CR}$, are elaborated and put into practice.
\end{abstract}

Keywords: Cardiac rehabilitation, Brazil, Poverty.

\section{RESUMEN}

Objetivo: Evaluar las barreras para la amplia implementación de la Rehabilitación Cardíaca que impiden que la población brasileña se adhiera a los programas de tratamiento y promover medidas públicas para cambiar esta realidad. Métodos: Este estudio es una revisión integradora de la literatura. Para la recolección de datos se utilizaron las plataformas: Acervo+, Biblioteca Virtual en Salud (BVS) y Pubmed y los descriptores en Ciencias de la Salud: "rehabilitación cardíaca", "Brasil" y "bajos ingresos" o "pobreza". Se incluyeron artículos completos en portugués, inglés y español, entre 2010 y 2021. La recolección se realizó en cinco etapas: identificación, selección, elegibilidad, inclusión y síntesis, recolección en diagramas de flujo y tablas para

1 Universidade Federal do Amazonas (UFAM), Manaus - AM. *E-mail: barbara.ihara@gmail.com 
análisis. Resultados: La búsqueda encontró 278 artículos. Después de leer el título y el resumen de los artículos, se excluyeron 250 artículos repetidos o atemáticos. Se seleccionaron 28 obras para lectura completa. Después del análisis, se incluyeron un total de quince artículos para esta revisión. Consideraciones finales: Es fundamental invertir en RC para la salud y la calidad de vida. Para ello, es necesario que se elaboren y pongan en práctica estrategias específicas, que aborden los principales puntos de dificultad para la implementación de la RC.

Palabras clave: Rehabilitación cardíaca, Brasil, Pobreza.

\section{INTRODUÇÃO}

As doenças cardiovasculares são uma das principais causas de morbimortalidade no Brasil e no mundo, causando um dispêndio financeiro e laboral muito grande para os governos. Felizmente, essas taxas estão em queda desde meados do século XXI, devido ao grande investimento na prevenção e elaboração de tratamentos multiprofissionais mais adequados. Um dos grandes aliados para a redução destes números (especialmente a prevenção secundária) é a Reabilitação Cardíaca (RC), que hoje abrange diversos patamares para evitar os prejuízos das cardiopatias (LIMA SC, 2016).

A partir da década de 1960, foram introduzidos no Brasil os programas de Reabilitação Cardíaca, sendo o cerne desse processo a prática de exercícios físicos prescritos de forma individualizada em relação à dose e frequência, com o objetivo de melhorar os componentes da aptidão física, aeróbicos e não aeróbicos, e dessa forma atuar na prevenção e controle das doenças cardiovasculares e de seus fatores de risco. Esse processo é considerado, hoje, um avanço considerável na restauração das funções físicas, psicossociais e individuais dos pacientes com algum tipo de doença coronariana pretérita. Além disso, é de fundamental importância na prevenção de novos episódios e na manutenção da saúde (CHAGAS AM, et al., 2019).

De acordo com Brown RA (1964), o Comitê de especialistas da Organização Mundial da Saúde (OMS) em reabilitação de pacientes com doenças cardiovasculares definiu a RC como um conjunto de atividades imprescindíveis para a manutenção das condições físicas, mentais e sociais de um paciente cardiopata, possibilitando seu retorno à comunidade com uma vida ativa e produtiva (BRAGA HDO, et al., 2015).

Com passar do tempo, viu-se que o conceito de RC abrangia muito mais do que o elaborado pela OMS. Além de auxiliar no tratamento de pacientes já acometidos por doenças, é um ato de promoção de saúde que, a guardar algumas particularidades, pode ser expandido para a população em geral. Por isso, atualmente, a $\mathrm{RC}$ passa a ser entendida como um processo contínuo de desenvolvimento e aplicabilidade de técnicas cada vez mais eficientes e adequadas à realidade de cada indivíduo (GUIMARÃES FADB, et al., 2015).

Diversos programas de RC foram criados no Brasil e no mundo. Com isso, diversos estudos vêm relatando que a reabilitação gera notáveis benefícios para os pacientes. Percebe-se uma melhora no quadro de morbidade e mortalidade, na qualidade de vida, no desempenho muscular e melhor resposta às atividades físicas. No entanto, apesar desses inúmeros benefícios, essa técnica é pouco utilizada e difundida na sociedade, com taxas de participação variando de $7,5 \%$ a $29 \%$, além de altas taxas de desistência, por volta de 50\% (ALMEIDA SANTOS LST, et al., 2017).

Nesse cenário, diversas são as barreiras que impedem a maior parte dos pacientes de ingressarem em programas de RC. Os percalços estão relacionados à família, ao quesito financeiro, ao estado de saúde do próprio paciente e, até mesmo, dificuldades de transporte. Outros fatores extremamente importantes são o nível de conhecimento, sendo que os indivíduos que têm mais aderência aos programas geralmente têm maior índice de escolaridade e sabem mais acerca do assunto sobre doenças cardiovasculares e RC. Além desses desafios, pode-se citar as baixas taxas de encaminhamento ao serviço de reabilitação que é um problema encontrado não apenas nos países em desenvolvimento como o Brasil, mas também em países considerados como de primeiro mundo, sendo resultado da própria opção do médico assistente em não fazer tal encaminhamento (CRUZ NEVES MS, et al., 2017).

Nesse sentido o motivo de parte dos médicos em orientar o paciente cardiopata a procurar o serviço de RC gera um questionamento do real conhecimento do profissional quanto aos benefícios terapêuticos da 
reabilitação. Ademais há uma grande dificuldade apresentada pelos financiadores de saúde, seja seguro privado, seja saúde pública, que ainda não compreendem a real relação do custo-benefício dos programas de RC no Brasil (LIMA SC, 2016).

Nesse ínterim, a localidade também influencia majoritariamente, visto que, no Brasil, os centros de reabilitação estão concentrados nas principais regiões do país e nas grandes metrópoles, deixando de lado as cidades com menor contingente populacional e menor aporte financeiro, a exemplo dos poucos programas de RC que se localizam na região Norte do país, mas que, evidentemente, necessita desses centros (LIMA SC, 2016).

No Brasil a distribuição dos centros de RC é altamente desproporcional, atualmente o cenário mais evidente que podemos afirmar é que os programas estão muito limitados aos centros metropolitanos do país. As alocações de serviços de reabilitação estão muito concentradas na região sudeste do país, enquanto isso as demais regiões representadas principalmente pelo Norte e Nordeste possuem um número extremamente escasso. Não bastasse essa questão, o número absoluto de centros de RC na região Amazônica é ainda mais baixo quando consideramos a informação de que em seu total, distribuído de maneira desuniforme para a região concentrada, eles não representam sequer um valor próximo do ideal que deveria existir para cobrir toda a população cardiopata com necessidade de reabilitação (BRITTO RR, et al., 2020).

Tendo em vista tais problemáticas, este estudo busca analisar na literatura a dificuldade de implementação e adesão da Reabilitação Cardíaca no Brasil, principalmente para pacientes de baixa renda, tomando por base o sucesso de outros países nesse processo e também na melhora da qualidade de vida do paciente.

\section{MÉTODOS}

Este estudo caracteriza-se por ser uma revisão de literatura integrativa a respeito das dificuldades que permeiam a RC. A revisão possui como questão norteadora o seguinte questionamento "Quais as principais dificuldades de reabilitar pacientes de baixa renda inseridos no Brasil?".

Para o levantamento bibliográfico foram utilizadas as plataformas Acervo+, Biblioteca Virtual em Saúde (BVS) e Pubmed, com auxílio dos descritores em ciências da Saúde (DeCS) em português e inglês, e suas combinações por uso dos operadores booleanos AND e OR: "reabilitação cardíaca", "Brasil" e "baixa renda" ou "pobreza".

Para a coleta e análise dos dados, determinou-se que seriam incluídos artigos científicos na íntegra de periódicos nacionais e internacionais disponíveis em português, inglês e espanhol, publicados entre os anos de 2010 a 2021 que abordavam como temática central a dificuldade da RC no país e estavam associados a pacientes de condição financeira vulnerável.

A coleta dos dados foi realizada em cinco etapas: identificação, seleção, elegibilidade, inclusão e síntese dos dados. Inicialmente, ocorreu a identificação dos dados por meio do levantamento nas bases de dados citadas, acessando diretamente as plataformas de cada base e utilizando os descritores para realizar a pesquisa, posteriormente, foi realizada a leitura do título e do resumo das publicações encontradas.

Em seguida, os artigos repetidos e que não se encaixavam nos critérios de inclusão a partir da leitura do título e resumo foram excluídos, os dados selecionados foram, então, lidos integralmente e aqueles que abordavam as questões relacionadas com a temática da presente pesquisa foram inseridos. Foram selecionados 278 artigos e, desses, quinze foram incluídos para revisão final.

Após a coleta dos dados, foram sintetizadas todas as informações em fluxograma (Figura 1) com as etapas definidas e em um quadro com as publicações de acordo com os autores, o periódico em que foi publicado, o ano de publicação, a base de dados ao qual foi extraído, a metodologia e os resultados.

Para a formulação do fluxograma foi utilizado o processador de texto Microsoft Word® 2020, para a elaboração do quadro foi utilizado o software Microsoft Excel® 2016 e para a organização das referências o gerenciador Mendeley ${ }^{\circledR}$ versão 1.19 .4 e. Foram selecionadas pesquisas referentes a RC em pacientes desprovidos de recursos financeiros com o intuito de conectar os artigos que abordam a temática e expor as informações de forma única. 


\section{RESULTADOS}

Realizou-se a busca nas bases de dados utilizando os descritores "reabilitação cardíaca" em associação com "baixa renda" ou "pobreza" e "Brasil". A pesquisa localizou 278 artigos, a grande maioria da plataforma BVS. Após leitura do título e resumo dos artigos excluímos um total de 250 que se repetiam entre as bases de dados, principalmente entre BVS e Pubmed, ou que não tinham conteúdo compatível com a temática central da pesquisa.

Dessa forma, foram selecionados 28 trabalhos para leitura na íntegra, priorizamos nessa etapa artigos que estivessem relacionados com a realidade da $\mathrm{RC}$ no Brasil e que, quando possível, voltados para a região norte do país, mas sem excluir publicações com dados julgados importantes para a interpretação da realidade de pacientes em vulnerabilidade.

Após a análise, foram incluídos um total de quinze artigos para a presente revisão, sendo 5 (33\%) da BVS, $9(60 \%)$ da Pubmed e $1(7 \%)$ do Acervo +. Desses, 6 (40\%) são estudo descritivos com delineamento transversal, $3(20 \%)$ são artigos de revisão, 2 (13,3\%) são coortes, 2 (13,3\%) são ensaios clínicos randomizados sendo um duplo-cego e o outro simples-cego. Além desses, houveram $1(6,7 \%)$ estudo qualitativo e $1(6,7 \%)$ artigo original de validação de uma escala de $R C$ para o Brasil.

A inclusão de estudos de revisão para esta pesquisa deu-se em virtude da insuficiente quantidade de publicações encontradas, dentro do período estabelecido, que permeassem a temática central da discussão. Posteriormente, todo o processo de coleta dos dados foi sintetizado em fluxograma explicativo de acordo com o proposto na metodologia (Figura 1).

Figura 1 - Seleção e coleta das publicações na amostra da revisão integrativa.

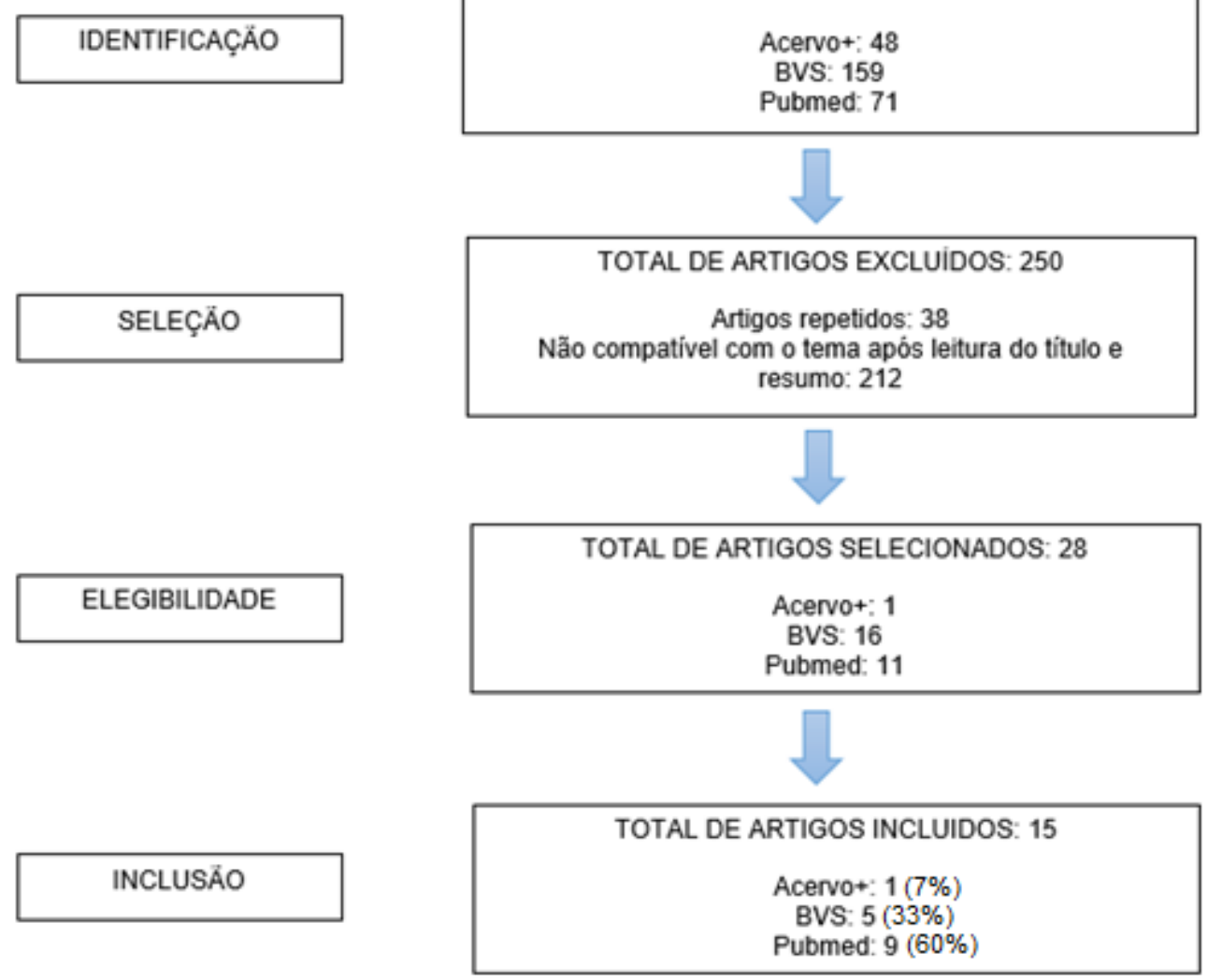

Fonte: Ihara BP, et al., 2021.

O Quadro 1 descreve os estudos selecionados para a presente revisão. Consta-se os autores de cada um, o periódico em que foi publicado, o ano de publicação, a base de dados ao qual foi extraído, a metodologia e os resultados. 
Quadro 1 - Síntese dos principais artigos selecionados.

\begin{tabular}{|c|c|c|c|}
\hline Autores e Ano / Periódico & Base de dados & Metodologia & Resultados \\
\hline $\begin{array}{l}\text { Araújo HVS, et al., 2017/ Revista } \\
\text { brasileira de enfermagem }\end{array}$ & Pubmed & $\begin{array}{l}\text { Estudo descritivo transversal. Realizado com } 75 \text { pacientes } \\
\text { submetidos à cirurgia de revascularização do miocárdio, } \\
\text { através da utilização de escore WHOQOL-Bref que avalia } \\
\text { qualidade de vida do paciente. }\end{array}$ & $\begin{array}{l}\text { Os pacientes apresentaram uma qualidade de vida regular no estudo, } \\
\text { porém com necessidade de melhora, principalmente quando } \\
\text { associada ao trabalho multiprofissional de reabilitação cardíaca. A } \\
\text { qualidade de vida também é pior em pacientes mais debilitados e com } \\
\text { baixa renda. }\end{array}$ \\
\hline $\begin{array}{l}\text { Borghi-Silva A, et al., 2014/ Progress } \\
\text { in cardiovascular diseases }\end{array}$ & BVS & $\begin{array}{l}\text { Artigo de revisão, com foco na } R C \text { no Brasil, abordando a } \\
\text { relação da } R C \text { com a prescrição de exercícios, as disparidades } \\
\text { entre as regiões do país, bem como os desafios de atendimento } \\
\text { e localização dos centros de reabilitação. }\end{array}$ & $\begin{array}{l}\text { Os programas formais de RC estão atualmente limitados a cidades } \\
\text { grandes do país, voltados para as metrópoles e insuficiente nas } \\
\text { regiões Norte e Nordeste. Além disso o menor nível de educação e o } \\
\text { elevado custo são colocados como barreiras explícitas para adesão } \\
\text { da RC. }\end{array}$ \\
\hline $\begin{array}{l}\text { Britto RR, et al., 2020/ Brazilian } \\
\text { journal of physical therapy }\end{array}$ & Pubmed & $\begin{array}{l}\text { Estudo transversal, para avaliar programas de } \mathrm{RC} \text { em todo o } \\
\text { mundo, comparando outros países com a realidade brasileira } \\
\text { acerca da disponibilidade e da qualidade do serviço prestado. }\end{array}$ & $\begin{array}{l}\text { A RC estava disponível em todas as regiões brasileiras, porém, com } \\
\text { uma concentração considerável na região sudeste do país. Havia } \\
\text { apenas um ponto de reabilitação cardíaca para cada } 99 \text { pacientes com } \\
\text { cardiopatia isquêmica. }\end{array}$ \\
\hline $\begin{array}{l}\text { Ghisi GLDM, el al., 2012/ Arquivos } \\
\text { Brasileiros de Cardiologia }\end{array}$ & BVS & $\begin{array}{l}\text { Tradução de escore. Analisa, traduz e adapta culturalmente a } \\
\text { escala de barreiras para reabilitação cardíaca (CRBS). A nova } \\
\text { versão CRBS foi testada em } 173 \text { pacientes cardiopatas e } \\
\text { analisada quanto a confiabilidade e validade de uso do escore } \\
\text { para pesquisar a dificuldade de reabilitação cardíaca no Brasil. }\end{array}$ & $\begin{array}{l}\text { A versão nova em português da CRBS possui confiabilidade. Tem a } \\
\text { importância de adaptar as questões e pesquisa para a realidade } \\
\text { brasileira. O processo de adaptação da linguagem ao ponto de vista } \\
\text { cultural aproxima a pesquisa do público alvo e aumenta a validação } \\
\text { do uso para pesquisa de dificuldade de reabilitação cardíaca. }\end{array}$ \\
\hline $\begin{array}{l}\text { Ghisi GLDM, et al., } 2013 \text { / Journal of } \\
\text { cardiopulmonary rehabilitation and } \\
\text { prevention }\end{array}$ & BVS & $\begin{array}{l}\text { Estudo descritivo com duas amostras constituídas por } 237 \\
\text { brasileiros e } 1434 \text { canadenses que foram comparadas } \\
\text { transversalmente. Foi utilizada a Escala de Barreiras de RC } \\
\text { (CBRS) para avaliar os fatores que afetam a adesão à RC. }\end{array}$ & $\begin{array}{l}\text { No geral, } 139(58,6 \%) \text { brasileiros e } 779 \text { (54,3\%) canadenses } \\
\text { respondentes estavam inscritos na RC. Para } 17 \text { das } 21 \text { barreiras, os } \\
\text { canadenses relataram como suas maiores dificuldades aspectos } \\
\text { pessoais, como o fato de se exercitar em casa, enquanto os brasileiros } \\
\text { identificaram a distância e o custo dos PRC. }\end{array}$ \\
\hline $\begin{array}{l}\text { Mair V, et al., 2013/ Einstein (São } \\
\text { Paulo) }\end{array}$ & Pubmed & $\begin{array}{l}\text { Estudo retrospectivo transversal com } 42 \text { pacientes participantes } \\
\text { de um programa de reabilitação cardíaca (RC) em um hospital } \\
\text { particular na região Sudeste do Brasil, por meio de duas } \\
\text { escalas, a de barreiras para a RC e a de disposição. }\end{array}$ & $\begin{array}{l}\text { As principais barreiras à adesão aos programas de RC foram } \\
\text { viagem/conflito de trabalho e problemas pessoais/familiares. Além da } \\
\text { baixa disposição, são alguns dos principais fatores de absentismo dos } \\
\text { programas de RC. }\end{array}$ \\
\hline $\begin{array}{l}\text { Nascimento IO, et al., } 2021 / \\
\text { Brazilian Journal Of Physical } \\
\text { Therapy }\end{array}$ & Pubmed & $\begin{array}{l}\text { Estudo qualitativo para avaliar a percepção de pacientes sobre } \\
\text { sua participação na reabilitação cardíaca (RC) através de } \\
\text { entrevista semi-estruturada com } 28 \text { participantes após } 2 \text { anos } \\
\text { de participação em dois modelos diferentes de RC no Brasil. }\end{array}$ & $\begin{array}{l}\text { Os pacientes dos dois modelos perceberam a longo prazo que a } \\
\text { participação na RC impacta positivamente seu conhecimento acerca } \\
\text { da enfermidade, bem como promoveu ganhos funcionais e melhorou } \\
\text { o bem-estar psicossocial. }\end{array}$ \\
\hline $\begin{array}{l}\text { Nesello PFT, et al., 2016/Open } \\
\text { Access Macedonian Journal Of } \\
\text { Medical Sciences }\end{array}$ & Pubmed & $\begin{array}{l}\text { Estudo de coorte histórica, com } 326 \text { pacientes submetidos a um } \\
\text { programa de reabilitação cardíaca (RC) na região Sul do Brasil, } \\
\text { subdivididos por idade, avaliando a taxa de abandono após } 48 \\
\text { sessões. }\end{array}$ & $\begin{array}{l}\text { A taxa de abandono total dos pacientes foi de } 38,6 \% \text {, os grupos de } \\
\text { Idoso-Jovem ( } 65 \text { a } 74 \text { anos) e Meia-Idade (75 a } 84 \text { anos) } \\
\text { apresentaram menor desistência quando comparado ao grupo Não- } \\
\text { Idoso (menor que } 65 \text { anos). Para uma RC efetiva, é necessário ajustar } \\
\text { os programas para uma melhor adesão das diferentes faixas etárias. }\end{array}$ \\
\hline
\end{tabular}




\begin{tabular}{|c|c|c|c|}
\hline Autores e Ano / Periódico & Base de dados & Metodologia & Resultados \\
\hline $\begin{array}{l}\text { Oldridge NB, et } \quad \text { al., } \\
\text { 2016/ International health }\end{array}$ & Pubmed & $\begin{array}{l}\text { Artigo de revisão, com abordagem sobre o custo e o custo } \\
\text { benefício da } \mathrm{RC} \text { em países de média e baixa renda, que inclui } \\
\text { e descreve as características do Brasil. }\end{array}$ & $\begin{array}{l}\text { A reabilitação cardíaca no Brasil é considerada custo-efetiva. No } \\
\text { entanto, o valor de custo não é considerado baixo, o que prejudica a } \\
\text { adesão de pessoas e estados com orçamentos limitados, havendo a } \\
\text { necessidade de programas de RC mais acessíveis. }\end{array}$ \\
\hline $\begin{array}{l}\text { Santos LSTA et al., 2017/ Acta } \\
\text { fisiátrica }\end{array}$ & BVS & $\begin{array}{l}\text { Estudo descritivo de corte transversal, que analisou } 79 \\
\text { participantes cardiopatas com idade superior a } 50 \text { anos em uma } \\
\text { cidade no nordeste brasileiro. Os pacientes foram informados } \\
\text { dos benefícios da RC e responderam um questionário } \\
\text { apontando as barreiras do programa. }\end{array}$ & $\begin{array}{l}\text { Dos paciente, } 81 \% \text { não tinham conhecimento dos benefícios que a RC } \\
\text { proporciona, } 63 \% \text { destacaram que a distância da residência até a } \\
\text { unidade é um problema, além disso, o alto custo com o transporte e a } \\
\text { não indicação da reabilitação pelo médico também foram citadas } \\
\text { como barreiras. }\end{array}$ \\
\hline $\begin{array}{l}\text { Sérvio TC, et al., 2019/ BMC health } \\
\text { services research }\end{array}$ & Pubmed & $\begin{array}{l}\text { Trata-se de um estudo descritivo multicêntrico, programas de } \\
\text { RC foram notificados em quatro regiões do estado de Minas } \\
\text { Gerais e } 41 \text { coordenadores de RC receberam uma pesquisa } \\
\text { para relatar a realidade do programa na prática. Realizando-se } \\
\text { uma diferenciação entre instiuições públicas e privadas. }\end{array}$ & $\begin{array}{l}\text { Foram identificados } 41 \text { programas de } \mathrm{RC} \text {, sendo } 21,9 \% \text { públicos. } \\
\text { Dezenove completaram a pesquisa. Os programas intra-hospitalares } \\
\text { foram localizados apenas em hospitais públicos. A principal barreira } \\
\text { para a participação da RC foi a falta de encaminhamento para o } \\
\text { serviço. }\end{array}$ \\
\hline $\begin{array}{l}\text { Sérvio TC, et al., 2018/ Brazilian } \\
\text { journal of physical therapy }\end{array}$ & BVS & $\begin{array}{l}\text { Trata-se de um estudo transversal, com dados de três coortes } \\
\text { (administradores de saúde, coordenadores de RC e pacientes), } \\
\text { no estado de Minas Gerais, Brasil. Os participantes } \\
\text { respodenram ao questionário de Corte-Bergoderi M et al. } \\
\text { (2013) que visa avaliar a situação dos programas de RC. }\end{array}$ & $\begin{array}{l}\text { Participaram trinta e dois }(35,2 \%) \text { administradores de saúde, } 16 \\
(28,6 \%) \text { provedores de RC e } 805 \text { pacientes cardíacos. Os } \\
\text { administradores reconheceram a importância do RC, mas também a } \\
\text { falta de recursos para entregá-lo; Os provedores de RC observaram } \\
\text { que há pouco encaminhamento por parte dos profissionais de saúde. } \\
\text { Pacientes que não foram inscritos na RC relataram barreiras } \\
\text { funcionais, econômicas e sociais. }\end{array}$ \\
\hline $\begin{array}{l}\text { Silva Chaves GS, et al., 2016/ } \\
\text { Brazilian journal of physical therapy }\end{array}$ & Pubmed & $\begin{array}{l}\text { Ensaio clínico randomizado duplo-cego, que conteve três } \\
\text { partes paralelas: RC abrangente (exercício + educação) versus } \\
\text { RC baseado em exercício versus controle de lista de espera } \\
\text { (sem RC), com o intuito de avaliar os efeitos da RC em } \\
\text { pacientes atentidos pela rede pública de saúde brasileira. }\end{array}$ & $\begin{array}{l}\text { Os resultados apresentaram-se como positivos e servem como } \\
\text { confirmação do benefício das estratégias de reabilitação, reforçando } \\
\text { que é fundamental o investimento por parte do governo e dos seguros } \\
\text { privados à } R C \text { em Belo Horizonte e em outras regiões do país. }\end{array}$ \\
\hline Silva Chaves GS, et al., 2019/ Heart & Pubmed & $\begin{array}{l}\text { Ensaio simples cego randomizado. Avalia os resultados da } \\
\text { reabilitação cardíaca com uso de grupo controle para } \\
\text { comparativo. Avaliou } 186 \text { pacientes com avaliação de resultado } \\
\text { no início da reabilitação e após } 6 \text { meses. }\end{array}$ & $\begin{array}{l}\text { A reabilitação cardíaca mostrou melhora clinica significativa no } \\
\text { paciente cardiopata sendo capacidade funcional aumentada, pressão } \\
\text { arterial controlada e o incremental Shuttle Wlak Test teve melhora nas } \\
\text { distâncias, os outros parâmetros avaliados não tiveram diferença } \\
\text { significativa com a RC. }\end{array}$ \\
\hline $\begin{array}{l}\text { Silva DB, et al., 2021/ Revista } \\
\text { Eletrônica Acervo Saúde }\end{array}$ & Acervo+ & $\begin{array}{l}\text { Artigo de revisão, que abrange as práticas de enfretamento às } \\
\text { doenças cardiovasculares (DCV), sejam elas de prevenção ou } \\
\text { de reabilitação. }\end{array}$ & $\begin{array}{l}\text { Muitas dificuldades são encontradas no que tange a instituição da } \\
\text { promoçãa em saúde frente ao avanço das DCV. Uma das principais é } \\
\text { a falta de orientação, de indicação e de otimização dos recursos para } \\
\text { os programas de reabilitação. }\end{array}$ \\
\hline
\end{tabular}

Fonte: Ihara BP, et al., 2021.

REAS | Vol.13(7) | DOI: https://doi.org/10.25248/REAS.e8372.2021 Página 6 de 10


Pode-se notar que a maior parcela dos artigos foi publicada em periódicos estrangeiros (8 ou $53 \%$ ) e a menor parte em periódicos brasileiros (7 ou 47\%) com pequena diferença. Em relação a distribuição das pesquisas por período de publicação, temos uma de 2012, duas de 2013, uma de 2014, três de 2016, duas de 2017, uma de 2018, duas de 2019, uma de 2020 e duas de 2021. Nota-se que a revista que mais publicou com relação ao tema foi o Brazilian Journal Of Physical Therapy com quatro publicações, seguida por Heart com duas publicações e as revistas restantes que continham um estudo cada.

\section{DISCUSSÃO}

Uma das principais problemáticas no âmbito da Reabilitação Cardíaca $(\mathrm{RC})$ é justamente a disponibilidade do serviço no país, principalmente no que concerne em oferecer tal tratamento de forma adequada para a população. Pode-se ver como é precária essa realidade nos estudos de Sérvio TC, et al. (2019), que demonstraram a falta de instituições públicas ou privadas, no Brasil, que disponibilizem essa medida para tratar dos efeitos de doenças cardiovasculares. Na maioria das cidades sequer há um programa de reabilitação bem estruturado e efetivo. Em contrapartida, países como o Canadá, também analisado na pesquisa, ofertam em maior quantidade essas clínicas de reabilitação, resultado de um investimento há décadas em prevenção primária e secundária.

Em comparação similar entre a realidade brasileira e o Canadá, a pesquisa de Ghisi GLDM, et al. (2013) denota que as principais barreiras frente à $R C$ no Canadá são a nível de paciente, ou seja, a sua adesão ou não, enquanto que no Brasil, isso se dá a nível de sistema de saúde. Os pesquisadores ainda delimitam que na realidade brasileira, esse fato ocorre em virtude dos poucos recursos disponibilizados aos centros de saúde para uma implantação efetiva e um maior incentivo à RC, citando como exemplo o fato de que o país possui salas de operação modernas capazes de realizar procedimentos cardíacos complexos, mas que os pacientes não possuem acesso à RC posteriormente.

Além disso, observa-se que a quantidade de Programas de Reabilitação Cardíaca (PRC) disponíveis e de qualidade satisfatória variam conforme cada localidade e seu índice de desenvolvimento humano (IDH) específico. O estudo de Sérvio TC, et al. (2019) atesta esse fato ao ilustrar a realidade dos PRC no estado de Minais Gerais, em que apenas 18 de 853 municípios apresentam tais programas. A pesquisa ainda subdividiu o estado em quatro regiões com características de IDH distintas e quantificou o número de programas em cada, identificando que as regiões norte e nordeste do estado, que possuem os menores IDH, não apresentaram nenhum PRC. Essa análise, quando realizada no âmbito nacional, pode ser corroborada pela produção de Borghi-Silva A, et al. (2014), que afirma que as regiões Norte e Nordeste do país, as mais desprovidas financeiramente, apresentam uma escassez evidente na disponibilidade de PRC.

Outra questão considerável seria a baixa adesão ao programa. Nota-se que em países de maior renda, as taxas de adesão tendem a serem favoráveis de acordo com a expectativa do país, no entanto, quando estão abaixo da expectativa, indicam que o principal fator de não adesão são de responsabilidade do paciente. A causa para tal fato é multifatorial e ainda é mal compreendida, a pesquisa de Sérvio TC, et al. (2019) sugere que esse fato possa ocorrer devido a muitos pacientes não saberem da importância ou não possuírem o interesse nesse tipo de prevenção, o que vale ressaltar a necessidade de haver educação em saúde para o público em geral que trate dos benefícios do tratamento e da melhora na qualidade de vida. Já em países de baixa renda ou em desenvolvimento, o estudo sugere que geralmente a causa da baixa adesão é justamente a falha por parte do sistema de saúde, no que tange ao oferecimento de clínicas e cursos de aperfeiçoamento dos profissionais de saúde.

Em complemento ao estudo citado e a temática da baixa adesão de pacientes a PRC, o estudo de Taylor $\mathrm{GH}$, et al. (2011), uma revisão sistemática que incluiu 8.842 pacientes, cita que entre outras causas para tal situação estão fatores sociodemográficos, psicológicos e médicos. Dentre os sociodemográficos, observa-se que pacientes mais jovens tendem a abandonar mais a RC, assim como mulheres. Nos fatores psicológicos, pacientes deprimidos apresentam maior dificuldade em concluir a reabilitação, enquanto que nos aspectos médicos, pacientes que já fizeram uma ou mais cirurgia cardíaca apresentam maior adesão, em contrapartida, 
os que são fumantes ativos ou possuem IMC elevado, tendem a não aderirem aos programas. Ainda assim, os autores concluem que os resultados são sugestivos, haja vista que os artigos analisados apresentaram muitas divergências, atestando que a causa da baixa adesão a PRC ainda precisa ser melhor estudada.

De acordo com Silva Chaves GS, et al. (2016), a RC tem papel fundamental na redução da pressão arterial, na diminuição de reincidências de doenças cardiovasculares e, provavelmente, uma redução na mortalidade. Tendo isso em vista, é importante notar que qualquer país que tenha deficiências nesse projeto vai ter consequências na área da saúde, com maior índice de internações, o que onera o sistema de saúde e afeta em muito a qualidade de vida da população.

É importante notar, também, que as dificuldades na popularização da Reabilitação Cardíaca passam também pela falha na divulgação e na busca pelos seus benefícios. Os estudos realizados acerca da temática geralmente englobam poucos pacientes e em poucos centros de saúde, dando espaço para o risco de viés e a dificuldade na generalização das informações encontradas. Por isso, os governos devem investir em estudos com maior contingente populacional, demonstrando que investimentos nessa área são imprescindíveis, tendo em vista que as doenças cardiovasculares se situam entre as que mais matam no Brasil e no mundo (SILVA DB, et al., 2021; LIMA SC, et al., 2016; SILVA CHAVES GS, et al., 2019).

Em uma análise atualizada da realidade brasileira quanto à demanda de vagas na $R C$, Britto $R R$, et al (2020) estimou que por ano são necessárias 500.000 vagas a mais para suprir as necessidades das pessoas acometidas por cardiopatias. Sem contar que o processo deve englobar várias etapas, que vão desde o encontro dos pacientes elegíveis, uma correta estratificação e avaliação do indivíduo, para adequar seus exercícios individualmente, até um acompanhamento periódico com a equipe multiprofissional de saúde, para reavaliações e orientações

No entanto, o Sistema Único de Saúde (SUS) é precário nesse quesito, focando apenas em algumas capitais, com as regiões mais longe dos grandes centros sendo esquecidas. São cerca de 75 programas desse tipo de reabilitação no Brasil, com cerca de 41 concentrados na Região Sudeste, com algumas divergências quanto ao número real a depender dos critérios de inclusão dos centros de tratamento (BRITTO RR, et al., 2020).

A diferença no tratamento da questão é evidente quando se tem noção que a grande parte dos estudos sobre esse tema se concentra nos estados das regiões Sul e Sudeste (NESELLO PFT, et al., 2016; BARROS AL, et al., 2014; LIMA SC, et al., 2016). Somado a isso, tem-se que o próprio conhecimento acerca do que seria os PRC entra como um dos fatores que atrapalham na adesão por parte dos pacientes, seja o conhecimento acerca da enfermidade em si, como também dos possíveis ganhos funcionais e melhora do bem-estar psicossocial envolvido. Além do mais, a falta da apresentação por parte do profissional de saúde também colabora para o triste cenário (NASCIMENTO IO, et al., 2021; MORAIS SANTOS TZ, et al., 2019).

Somado a tal cenário, as próprias limitações dos pacientes são fatores relevantes para a baixa adesão à RC. Sob o ponto de vista epidemiológico, tem-se que o principal público-alvo da reabilitação seriam idosos e que, geralmente, são de baixa renda e de baixa escolaridade. Isso implica em desconhecimento acerca do tema e dos benefícios adquiridos, além de dificuldades de locomoção para frequentar as sessões. Somado a isso, tem-se também a descontinuidade no tratamento medicamentoso, que é comum de ocorrer, levando a um aumento na probabilidade de outros eventos cardiovasculares (ARAÚJO HVS, et al., 2017; BERRY JRS e CUNHA AB, 2010; OLDRIGE NB, et al., 2016).

Os estudos de Santos LSTA, et al. (2017), Silva SB, et al. (2021) e de Mair V, et al. (2013) acrescentam outros fatores limitantes à adesão de pacientes aos PRC como o custo com a mobilidade urbana, que dificulta ainda mais a locomoção até o centro especializado, a não indicação do médico por achar o serviço desnecessário, a possibilidade de se exercitar em casa ou na comunidade, a baixa disposição de muitos pacientes e problemas relacionados aos aspectos pessoais, familiares e laborais.

Algumas soluções estão sendo buscadas para sanar as falhas na implementação dos PRC. O estudo de Borghi-Silva A, et al. (2014) considera que, apesar dos programas serem concentrados em grande parte nos principais hospitais federais e estaduais no Rio-São Paulo, há como expandir para as regiões mais distantes 
e com menor aporte financeiro, com a implementação de ginásios e treinamento dos integrantes das equipes multiprofissionais de saúde, criando meios adaptados ao ambiente em que se vive, com custo reduzido e que tenha efetividade de fato na mudança da qualidade de vida do paciente. Apesar disso, a população que reside em zona rural fica desemparada nessa questão e mais estudos devem ser fomentados para solucionar a problemática, além de esforços da esfera federal, estadual e municipal.

Reconhecendo a importância da RC, a Agência Nacional de Saúde Complementar (ANS) resolveu em 2011 tornar a Reabilitação Cardíaca como procedimento obrigatório e caso esse não seja oferecido pelo plano de saúde, deve ser reembolsado ao paciente. Isso demonstra o reconhecimento por parte das empresas do ramo da saúde sobre a importância de tais procedimentos, apesar dos problemas de sua implementação e adesão (BRASIL, 2011).

Entre outras possibilidades para tentar implementar os PRC, em especial, para aqueles pacientes que não tem acesso aos centros de RC em sua cidade, as novas tecnologias surgem como uma possível alternativa, haja vista que programas de monitoramento e a educação a distância, bem como a telessaúde, podem ser fornecidos como uma forma mais simples de acesso a RC. Para os pacientes com um acesso mais escasso, outras estratégias podem ser utilizadas visando à adesão aos PRC, quando disponíveis. Entre elas podemse citar os PRC à domicílio e o incentivo à utilização do Tratamento Fora do Domicílio (TFD) para usuários do SUS (SÉRVIO TC, et al., 2019; SANTOS LSTA, et al., 2017).

\section{CONSIDERAÇÕES FINAIS}

Esta revisão permitiu analisar que há uma quantidade escassa de publicações referentes às principais dificuldades de implementação e adesão da RC no Brasil. Nos estudos analisados, evidenciou-se que é de fundamental importância investir e disseminar a RC visando à saúde e qualidade de vida de pacientes cardiopatas. Esta produção tem como finalidade embasar o Poder Público no que tange à RC, reconhecendo suas dificuldades de implementação a fim de contorná-las. Além disso, é importante conscientizar os pacientes sobre a importância desse tipo de tratamento. Por fim, sabe-se que pela diversidade socioeconômica no país, é necessário buscar maneiras replicáveis em todos os estados para aderirem à RC, tendo a modalidade domiciliar como uma alternativa de vencer essas dificuldades e possibilitar aos pacientes de baixo e moderado risco o acesso ao tratamento em cidades nas quais não se tem centros especializados.

\section{REFERÊNCIAS}

1. ALMEIDA SANTOS LST, et al. Barreiras da reabilitação cardíaca em uma cidade do nordeste do Brasil. Acta fisiátrica, 2017; 24(2): 67-71.

2. ARAÚJO HVS, et al. Quality of life of patients who undergone myocardial revascularization surgery. Revista brasileira de enfermagem, 2017; 70: 257-264.

3. BARROS AL, et al. Diferentes barreiras para reabilitação cardíaca. Rev Bras Cardiol, 2014; 27(4): $293-298$.

4. BERRY JRS, CUNHA AB. Avaliação dos efeitos da reabilitação cardíaca em pacientes pós-infarto do miocárdio. Rev Bras Cardiol, 2010; 23(2): 1-12.

5. BORGHI-SILVA A, et al. Current trends in reducing cardiovascular disease risk factors from around the world: focus on cardiac rehabilitation in Brazil. Progress in cardiovascular diseases, 2014; 56(5): 536-542.

6. BRAGA HDO, et al. Protocolo de samba brasileiro para reabilitação cardíaca. Revista Brasileira de Medicina do Esporte, 2015; 21(5): 395-399.

7. BRASIL. ANEEL. Resolução Normativa №262 de 01 de Agosto de 2011. Atualiza o Rol de Procedimentos e Eventos em Saúde, 2011; 1(1): $1-11 . \quad$ Disponível em: https://bvsms.saude.gov.br/bvs/saudelegis/ans/2011/res0262_01_08_2011.html\#: :text=Atualiza\%200\%20Rol\%20d e\%20Procedimentos, 11\%20de\%20janeiro\%20de\%202010.\&text=1%20A\%20presente\%20Resolução\%20Normativ a,11\%20de\%20janeiro\%20de\%202010.

8. BRITTO RR, et al. Cardiac rehabilitation availability and delivery in Brazil: a comparison to other upper middle-income countries. Brazilian journal of physical therapy, 2020; 24(2): 167-176.

9. BROWN RA. Rehabilitation of patients with cardiovascular diseases: report of a WHO expert committee. World Health Organ Tech Rep Ser, 1964; 270: 3-46.

10. CHAGAS AM, et al. Reabilitação cardíaca fase I: uma revisão sistemática. ASSOBRAFIR Ciência, 2019: 7(3): 51-60. 
11. GHISI GLDM, et al. Barriers to cardiac rehabilitation use in Canada versus Brazil. Journal of cardiopulmonary rehabilitation and prevention, 2013; 33(3): 173-179.

12. GHISI GLDM, et al. Desenvolvimento e validação da versão em português da Escala de Barreiras para Reabilitação Cardíaca. Arquivos Brasileiros de Cardiologia, 2012; 98(4): 344-352.

13. GUIMARÃES FADB, et al. Reabilitação Cardíaca, tratamento e prevenção: Revisão bibliográfica. Revista Movimenta, 2015; 8(1): 50-59.

14. LIMA SC, et al. Conhecimento sobre doença arterial coronariana e barreiras para adesão à reabilitação cardíaca. ASSOBRAFIR Ciência, 2016; 7(2): 45-56.

15. MAIR V, et al. Avaliação da aderência ao programa de reabilitação cardíaca em um hospital particular geral. Einstein (São Paulo), 2013; 11(3): 278-284.

16. MORAIS SANTOS TZ, et al. Sistemas de saúde e percepção de barreiras para admissão e aderência em programas de reabilitação cardíaca. Acta fisiátrica, 2019; 26 (2).

17. NASCIMENTO IO, et al. A qualitative study of patient's perceptions of two cardiac rehabilitation models. Brazilian Journal Of Physical Therapy, 2021; 1 (1).

18. NESELLO PFT, et al. Treatment of the Aged Patients at a Large Cardiac Rehabilitation Center in the Southern Brazil and Some Aspects of Their Dropout from the Therapeutic Programs. Open Access Macedonian Journal Of Medical Sciences, 2016; 4(4): 654-660.

19. OLDRIDGE NB, et al. Cardiac rehabilitation in low-and middle-income countries: a review on cost and costeffectiveness. International health, 2016; 8(2): 77-82.

20. Organização Mundial da Saúde. Rehabilitation of patients with cardiovascular diseases. Report of a WHO expert committee. World Health Organ Tech Rep Ser, 1964; 270:3-46.

21. SANTOS LSTA, et al. Barreiras da reabilitação cardíaca em uma cidade do nordeste do Brasil. Acta fisiátrica, 2017; 24(2): $67-71$.

22. SÉRVIO TC, et al. Availability and characteristics of cardiac rehabilitation programs in one Brazilian state a crosssectional study. Brazilian journal of physical therapy, 2018; 22(5): 400-407.

23. SÉRVIO TC, et al. Barriers to cardiac rehabilitation delivery in a low-resource setting from the perspective of healthcare administrators, rehabilitation providers, and cardiac patients. BMC health services research, 2019; 19(1): 1-10.

24. SILVA CHAVES GS, et al. Effects of comprehensive cardiac rehabilitation on functional capacity and cardiovascular risk factors in Brazilians assisted by public health care: protocol for a randomized controlled trial. Brazilian journal of physical therapy, 2016; 20: 592-600.

25. SILVA CHAVES GS, et al. Effects of comprehensive cardiac rehabilitation on functional capacity in a middle-income country: a randomised controlled trial. Heart, 2019; 105(5): 406-413.

26. SILVA DB, et al. Enfrentamento das doenças cardiovasculares na atenção básica: revisão integrativa da literatura. Revista Eletrônica Acervo Saúde, 2021; 13(2): e5636.

27. TAYLOR GH, et al. Medical, psychological, and sociodemographic factors associated with adherence to cardiac rehabilitation programs: a systematic review. Journal of Cardiovascular Nursing, 2011; 26(3): 202-209. 